\title{
Irish children's food and beverage portion sizes: a qualitative study of parents' views and practices
}

\section{Abstract}

Food portion sizes routinely consumed by children have increased over time and consumption of larger portion sizes is associated with higher weight status. Parents often have the critical role of deciding portion sizes of foods to serve their children. It is not well understood, however, how parents decide the amount of food to serve. This research aimed to investigate parental views on portion sizes of foods and beverages served to children.

A purposive sample of preschools and primary schools geographically located in either urban or rural areas of Northern Ireland and the Republic of Ireland, and classified as either high or low deprivation were recruited. Selected schools were invited to facilitate this research. Parents with at least one child aged 2-12 years attending a recruited school were then invited to participate in a focus group. A maximum of three groups were scheduled with interested parents at each school. One researcher moderated the focus groups, assisted by another researcher in all groups where possible. A semi-structured discussion guide was followed, using probes to stimulate discussion. The guide explored practices in portioning foods and beverages for children, factors influencing portioning and views on portion sizes of foods and beverages served to children. Discussions were audio-recorded, transcribed verbatim and data was analysed thematically. Nvivo software was used to manage the coded data.

Focus groups $(\mathrm{n}=24)$ ranging in size from 3-12 parents were conducted across 19 schools. The response rate from parents at recruited schools ranged from $1-16 \%$. A total of 144 parents $(86 \%$ female, mean age $46 \mathrm{y}$ ) participated, of which $60 \%$ had a child attending a high deprivation school and $64 \%$ had a child attending a rural school. Factors influencing parental portioning included seven themes comprised of sub-themes: parent knowledge (portion size knowledge, serving a learned amount), parent motivations (ensure child is fed, encourage healthier food), child-related factors (appetite, body size, food preferences, eating patterns), family influence (spouses and grandparents serving), food retail and other settings (restaurants, takeaways, special occasions), socio-cultural influences (parent childhood experiences, parents' portion size norms) and portioning resources used (serve ware, utensils, hand sizes).

Parents' main concern regarding the amount they feed their children was to ensure that they are fed a sufficient amount to feel full. Parents learned this amount through experience and felt it was specific to each child's appetite. These findings will inform future interventions and information campaigns to help parents understand child portion sizes.

\section{Conflict of Interest}

There is no conflict of interest 